\title{
98
}

\section{Task analysis for collaborative work}

\author{
Andrew Monk and Leon Watts \\ Department of Psychology, University of York, York, YO1 5DD, U.K. \\ Email: [am1 I law4]@york.ac.uk
}

\begin{abstract}
Collaborative work requires all participants to synchronise their separate activities through their awareness of each other. This paper proposes a diagrammatic scheme for reasoning about this synchronisation for the purpose of configuring communication technologies. The Comms Usage Diagram (CUD) was developed in the course of three field studies of medical teleconsultation.
\end{abstract}

KEYWORDS Task analysis, CSCW, telemedicine, collaborative work, communication technology.

\section{INTRODUCTION}

The diagrammatic representation described here was developed in the course of three studies of medical teleconsultation. The sites visited used videophone technology to connect the occupants of a treatment room in a primary care centre with a consultant in a hospital. A typical consultation would involve talking about a patient's problem and viewing some visual information such as an X-ray. A striking feature of these consultations was the role played by peripheral parties. When the GP was talking to the consultant the patient could generally overhear and interject. Patients are often accompanied by a relative, there may also be a nurse or technician present. Again, these peripheral participants are aware of what is going on and may take some part in the activity being undertaken. Of course, the communication technologies used restrict the information available to the remote parties. For examplc, the consultant will only be able to see whoever is sitting directly in front of the videophone. It is thus incumbent on the GP to make sure that the consultant is aware of the presence of everyone in the treatment room at the start of the consultation. The consultant is then able to use language that is appropriate to the situation.

This sensitivity to the identity and awareness of one's audience is typical of collaborative work (Clark \& Schaefer, 1989). When deciding on how to best configure communication facilities to support collaboration one needs to reason about the requirements of different activities and phases of the work in this regard. This then is the purpose of the representation described here, to reason about collaborative work and the potential impact of communications technologies when configuring those technologies for some particular task.

A common abbreviation in engineering for communications technologies is "comms" hence the graphical representation proposed will be referred to as a Comms Usage Diagram or CUD. Graphical representations are commonly used in task analysis (Kirwan \& Ainsworth, 1992) to summarise and reason about work and the CUD is in this tradition. An analyst will use any information available to come to some understanding of the work. Because of the diversity of these sources of information, e.g., interviews and direct observation, some common representation is required to integrate and reason about the understanding acquired.

\section{A SAMPLE CUD}

Figure 1. is part of the CUD created to reason about the work at one field study site. There are two locations a Minor Treatment Centre staffed by nurse practitioners in London and an accident and emergency department in Belfast. The first two columns indicate the activities going on at each of these locations and the people who are present.

Primary and peripheral participants:- Primary participants are named with the activity. For example, there is an activity where the nurse practitioner and consultant "discuss case". Here the primary participants 
are the nurse practitioner and the consultant but the patient and a relative of the patient are also present in the treatment room. They are considered as peripheral participants and represented by "(P, R)".

Sequence:- The CUD can be read from top to bottom giving the approximate order of events. Task phases are relatively invariant. These are signified by horizontal rules across the full width of the CUD (see Figure 1.) The sequence of activities within a task phase is more varied and does not have to be made explicit. Order is implied through the content of the activities and where joint activities have a strong sense of initiation or causation, through the use of single headed arrows. In the first task phase in Figure 1. the ordering of the events, placing a call and fetching the consultant is implied by the single headed arrows. The activities of "greeting" and "discussing" use double headed arrows. The ordering of these events is implied by their content.

Mediation and its effects:- Comms resources are listed in the first place at the same horizontal level as the activity to which they refer. Activities given further down the CUD are taken to inherit these. Any changes to the configuration are then noted next to the activity at which the change was made. The rightmost column records the relevant "effects": whether a point is positive or negative and the participant affected. They vary considerably in scope. The alternative course of action, had the videophone not been available, would have been to refer the patient on. Figure 1. notes an advantage for the nurse practitioner of a reduction in paper work relative to this alternative. This is an advantage of broad scope brought about by the new way of working that is made possible by the technology. At the other extreme there are points made that apply only to the operation of the mobile camera.

\section{DISCUSSION}

The notes made in the rightmost column of the CUD are the major reason for performing the analysis. They will normally originate in a comment from an informant. What the CUD adds is the possibility to locate the advantage or disadvantage more precisely within the work and to find other places in the work that could be affected similarly.

The CUD is an effective tool for reasoning about the technological support of collaborative work through communication technologies. By using this representation we were able to summarise and reason about the data given by our informants. It was possible to characterise how communication occurred between all participants in a way that could be made accessible to those informants so that they could comment on our conclusions. The account was compact, two sides of A4 for two sites and one for the other. By using this representation we were able to make strong recommendations about the use of video and audio technology for teleconsultation (Watts \& Monk, 1997).

\section{REFERENCES}

Clark, H. H., \& Schaefer, E. R. (1989). Contributing to discourse. Cognitive Science, 13, 259 - 294.

Kirwan, B. \& Ainsworth, L. K. (1992). The task analysis guide. London: Taylor and Francis.

Watts, L. A. \& Monk, A. F. (1997). Telemedical consultation: task characteristics. In $C H I^{\prime} 97$, New York: ACM.

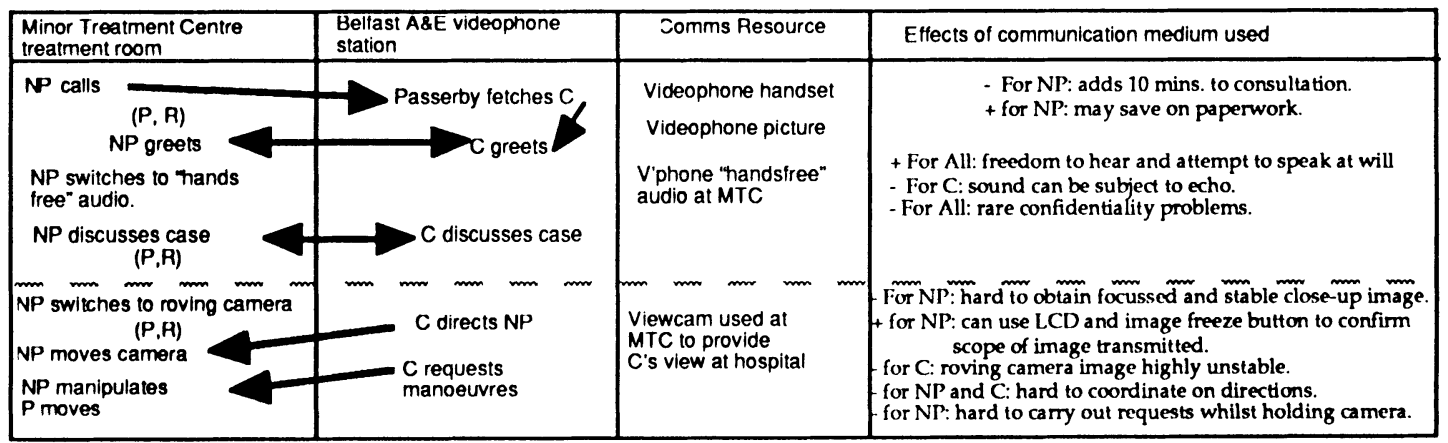

Figure 1. A CUD fragment. NP= Nurse Practitioner; $C=$ Consultant; $P=$ Patient; $R=$ Relative. 